\title{
Comparative efficacy of targeted maintenance therapy for newly diagnosed epithelial ovarian cancer: a network meta-analysis
}

This article was published in the following Dove Press journal: Cancer Management and Research

\author{
Xiaoyu $\mathrm{Xu}^{\prime}$ \\ Songcheng Yin $^{2,3}$ \\ Hongling Guo' \\ Mengxiong $\mathrm{Li}^{\prime}$ \\ Zhirong Qian ${ }^{4}$ \\ Xiaohui Tian' \\ Tian $\mathrm{Li}^{\prime}$
}

'Department of Gynecology, The Seventh Affiliated Hospital of Sun Yat-sen University, Shenzhen, People's Republic of China; ${ }^{2}$ Center for Digestive Disease, The Seventh Affiliated Hospital of Sun Yat-sen University, Shenzhen, People's Republic of China; ${ }^{3}$ Department of Surgical Oncology, First Affiliated Hospital of China Medical University, Shenyang, People's Republic of China; ${ }^{4}$ Scientific Research Center, The Seventh Affiliated Hospital of Sun Yat-sen University, Shenzhen, People's Republic of China
Correspondence: $\mathrm{Tian} \mathrm{Li}$

Department of Gynecology, The Seventh Affiliated Hospital of Sun Yat-sen

University, 628 Zhenyuan Road,

Shenzhen, People's Republic of China

Tel +8607558 1206773

Fax +8607558 I20 6773

Email litianlucky@126.com
Background: The number of published randomized clinical trials (RCTs) using targeted maintenance therapy for newly diagnosed epithelial ovarian cancer is increasing. Our objective was to evaluate the comparative effectiveness of each maintenance therapy using a network meta-analysis.

Materials and methods: A systematic search for RCTs was conducted using Medline, Embase, and CENTRAL databases followed by a Bayesian network meta-analysis. The primary outcome was progression-free survival (PFS) and the secondary outcome was overall survival (OS). Pooled hazard ratios (HRs) with $95 \%$ credible intervals $(95 \% \mathrm{CrIs})$ were used to estimate outcomes.

Results: A total of 11 RCTs involving 6631 patients were included. Network meta-analysis showed that pure maintenance therapy with pazopanib resulted in a significantly better PFS compared with placebo (HR, 0.77; 95\% CrI, 0.65-0.92). Bevacizumab-throughout treatment was also associated with a better PFS (HR, 0.76, 95\% CrI, 0.69-0.84). However, anti-CA-125 monoclonal antibodies (abagovomab and oregovomab) showed no significant survival benefit. Moreover, combined analysis showed that targeted-throughout was not significantly superior to pure targeted maintenance therapy for PFS and OS. Stratified analysis showed paralleled results with no significant difference between pazopanib pure maintenance and bevacizumab-throughout treatments.

Conclusion: Our study showed a survival advantage conferred by pazopanib and bevacizumab as maintenance therapy in newly diagnosed epithelial ovarian cancer. Further clinical trials are essential to both determine the effect of bevacizumab in the maintenance stage and identify the specific subgroup(s) that benefit.

Keywords: targeted treatment, maintenance therapy, ovarian cancer, network meta-analysis

\section{Introduction}

Ovarian cancer represents the six most common female malignancy in the world and has the highest mortality rate among all gynecologic cancers. ${ }^{1}$ Aggressive surgery followed by platinum/taxane-based chemotherapy is the standard treatment option. $^{2}$ But long-term survival remains low (10-30\%), mainly because of disease recurrence and progression. ${ }^{3}$ Maintenance therapy, a strategy to delay progression or recurrence by killing residual slowly dividing cells, has been a focus in recent years. However, multiple previous trials of cytotoxic agents in the maintenance setting have not shown a clear survival advantage but rather a significant increase in risk for long-term chemotherapy-related cumulative toxicity. ${ }^{4-6}$

Based largely on the GOG-0218 and ICON7 trials, which demonstrated high efficacy and less adverse reactions compared with cytotoxic agents, tumor molecular 
targeted therapy has drawn much attention in regard to maintenance therapeutic applications for ovarian cancer. ${ }^{7,8}$ Targeted drugs can be divided into several categories according to their target, some of which include: anti-epidermal growth factor receptor (EGFR) drugs such as erlotinib and gefitinib; drugs acting on the vascular endothelial growth receptor (VEGFR) such as bevacizumab, pazopanib, and sorafenib; and monoclonal antibody drugs specific for CA125 such as oregovomab and abagovomab. At present, there are two main therapeutic strategies for targeted maintenance therapy in newly diagnosed ovarian cancer: maintenance therapy after first-line chemotherapy (pure targeted maintenance treatment) or combined with chemotherapy and longterm maintenance (targeted-throughout treatment). ${ }^{7,9}$ Moreover, different target maintenance drugs have performed inconsistently in regard to survival outcomes. $7,9,10$ The existing randomized controlled trials (RCTs) and conventional meta-analyses have only partially captured evidence for treatment strategies for newly diagnosed ovarian cancer; the comparisons among treatment strategies remain unknown.

Previous studies have reported targeted maintenance therapy for ovarian cancer. ${ }^{11,12}$ However, the optimal strategy for this remains unclear. In the current study, we present a network meta-analysis comparing the relative efficacy of all available treatments for target maintenance treatment in newly diagnosed epithelial ovarian cancer. The advantage of this method over conventional metaanalysis is that it allows the assessment of treatments that have not been compared directly or indirectly in trials. $^{13,14}$

\section{Methods}

This meta-analysis was performed in agreement with the recommendations of the preferred reporting items for systematic reviews and meta-analyses (PRISMA guidelines).

\section{Search strategy and selection criteria}

Medline, Embase, and the Cochrane Library were systematically searched (from the date of inception to 30 March 2018) using methods described previously. ${ }^{15,16}$ The search strategy consisted of medical subject headings $(\mathrm{MeSH})$ terms and keywords for epithelial ovarian cancer and targeted therapy. Simultaneously, the reference lists of related articles from studies enrolled during our initial search were retrieved manually. Two investigators (XYX and SCY) each performed a complete literature search independently.
Studies had to meet the following inclusion criteria: (1) prospective phase II or III RCTs; (2) patients with pathologically proven newly diagnosed epithelial ovarian cancer; (3) patients were pretreated with systematic chemotherapy or cytoreductive surgery; (4) the intervention group used single-agent targeted maintenance therapy, and the control group used the matching placebo; (5) available hazard ratios (HRs) could be calculated for outcomes. Patients with recurrent ovarian cancer were excluded in our study. Additionally, we extracted the updated data for trials which were reported multiple times.

\section{Data extraction and quality assessment}

Relevant data elements were extracted from each study, including patient demographics (eg, region, race), baseline characteristics (eg, age, FIGO stage, median follow-up), and study features (eg, first author, sample size, year of publication, targeted agent, outcome measures). The Cochrane risk-of-bias tool was used to evaluate the quality of the original RCTs, taking into account randomization, blindness, incomplete outcome data, and any other potential source of bias. Two reviewers (XYX and SCY) independently conducted the data extraction and quality assessment.

\section{Statistical analysis}

The primary outcome was progression-free survival (PFS) and the secondary outcome was overall survival (OS). For time-to-event outcomes, HR with $95 \%$ confidence interval (CI) was the preferred outcome measure. If this was unavailable, it was calculated from the survival curves using established methods. ${ }^{17}$ For direct meta-analysis, Cochran's $Q$ statistic and $I^{2}$ tests were used to assess the heterogeneity among the studies. If the result was $P<0.10$ or $I^{2}>50 \%$, the random-effects model was conducted. In all other cases, the fixed effects model was implemented.

For indirect comparisons between regimens, a Bayesian network meta-analysis using Markov chain Monte Carlo methods and the GeMTC package in R (https://drugis.org/ software/r-packages/gemtc) was conducted. ${ }^{18}$ The transitivity assumption depended on a common treatment (placebo), which was comparatively consistent among all the included RCTs. Treatment effects were described by posterior means with corresponding 95\% credible intervals (CrIs). The probability of each regimen being the optimal was used to provide a rank of treatments. The consistency test was assessed by comparing the results generated from the network metaanalysis with direct pairwise comparisons. Potential 
publication bias was assessed by Begg's test and funnel plots. The pair-wise meta-analysis and network meta-analysis were performed with Stata 14.0 (StatCorp LLC, College Station, TX, USA) and R 3.3.3, respectively. All statistical tests were 2-sided.

\section{Results}

\section{Description of the included studies}

A total of 11 RCTs involving 6631 patients were included in this meta-analysis after completion of the literature search (summarized in Figure 1). ${ }^{7-10,19-25}$ There were two targeted drug delivery strategies among the eligible RCTs. The trial designs for six studies were purely targeted maintenance therapies, in which patients received targeted therapy after first-line treatment. Five studies conducted targeted-throughout treatment, in which patients received chemotherapy plus concurrent targeted regimen followed by single-agent targeted drug during the maintenance period. Patients received nine different targeted therapies which included tanomastat, sorafenib, abagovomab, pazopanib, oregovomab, erlotinib, bevacizumab, lonafarnib, and enzastaurin (Table 1). The sample size in each of the eligible studies varied from 85 to 1528 . Because all eligible studies included patients undergoing debulking surgery, some patients had residual lesions. For pure maintenance studies, most patients received first-line chemotherapy with no evidence of disease (eg, computed tomography and CA-125 levels) on general examination.

\section{Network meta-analysis Pure targeted maintenance treatment}

The network meta-analysis incorporated six direct comparisons for pure targeted maintenance designs; a diagram is shown in Figure 2A. Among the six comparisons, pazopanib was the only treatment with a significant improvement in PFS compared with placebo (HR, 0.77; 95\% CrI, 0.650.92); tanomastat, sorafenib, abagovomab, oregovomab, and erlotinib had no significant PFS benefit (Figure 2B).

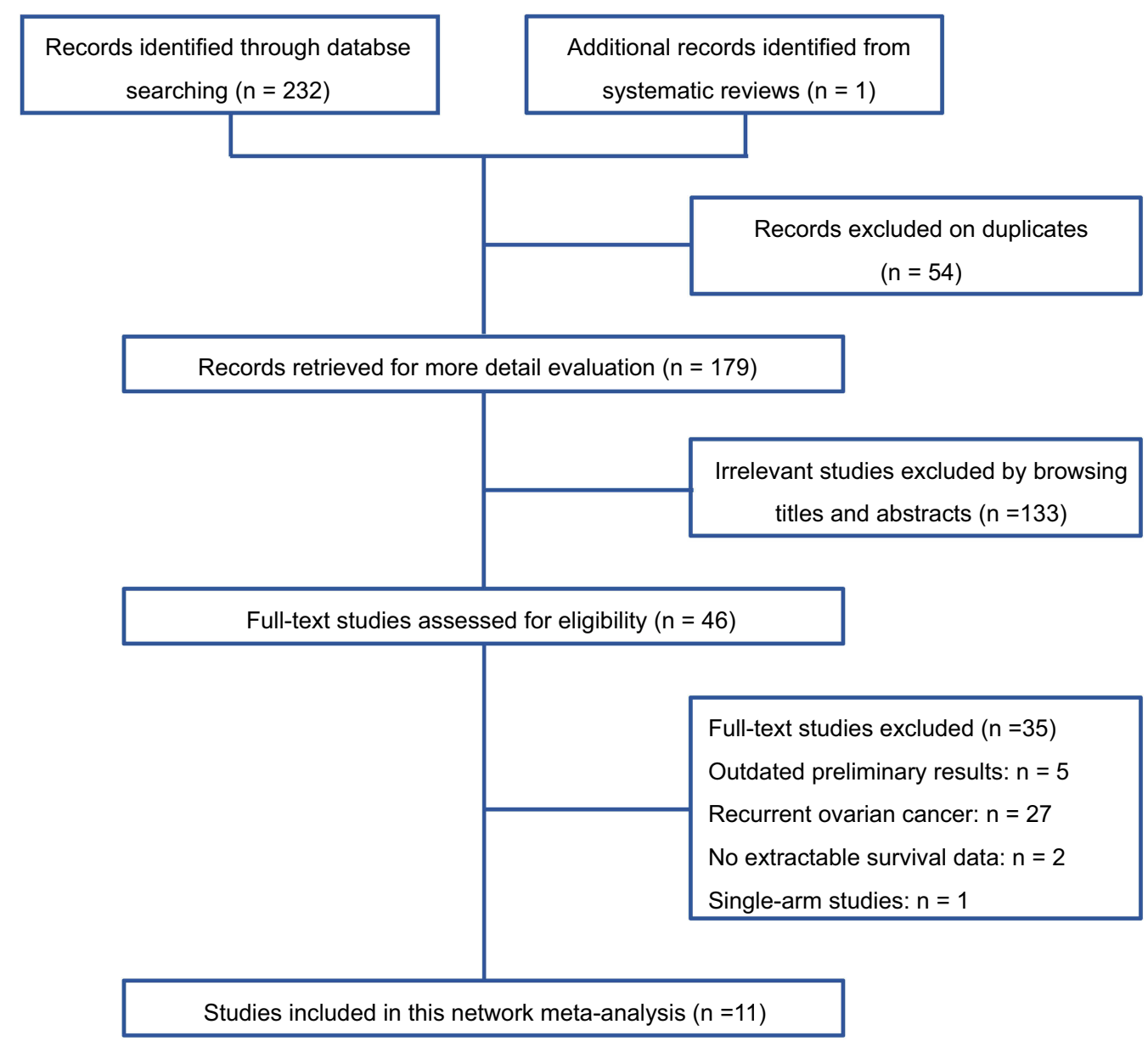

Figure I PRISMA flowchart of the study selection process. 


\begin{tabular}{|c|c|c|c|c|c|c|c|}
\hline 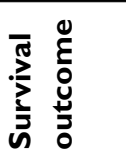 & $\begin{array}{l}\text { Oั } \\
\text { 뜸 }\end{array}$ & $\stackrel{\breve{L}}{\underline{a}}$ & $\begin{array}{l}\check{0} \\
\text { 똠 }\end{array}$ & 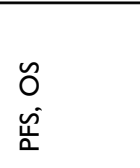 & 씸 & 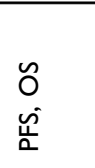 & 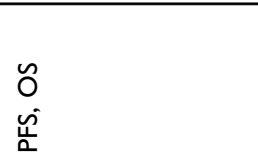 \\
\hline 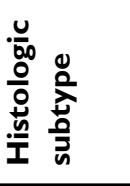 & 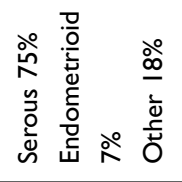 & 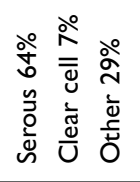 & \multicolumn{5}{|c|}{ 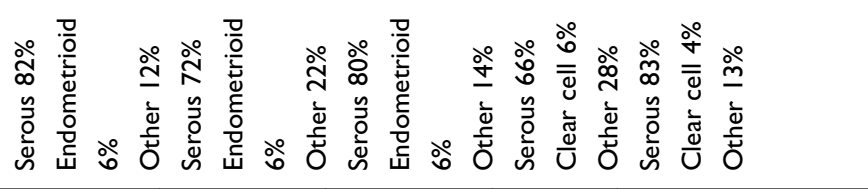 } \\
\hline ๕్ّ & \multicolumn{5}{|c|}{ 旁 } & $\S$ & 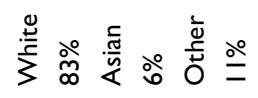 \\
\hline 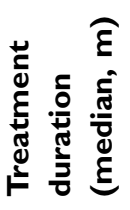 & స్ & $\bar{\forall}$ & $\stackrel{\cong}{=}$ & $\underset{\infty}{\sigma}$ & 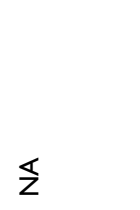 & $\bar{\infty}$ & $\mathbb{Z}$ \\
\hline 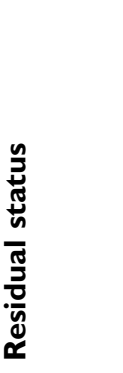 & 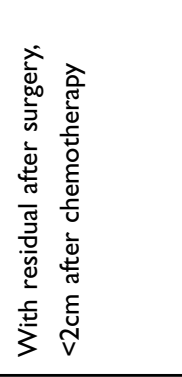 & 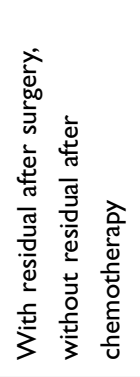 & 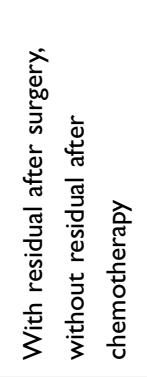 & 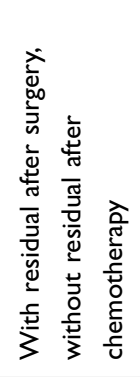 & 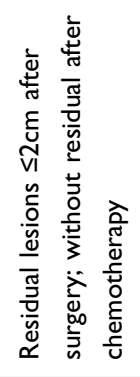 & 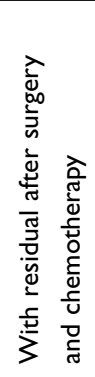 & 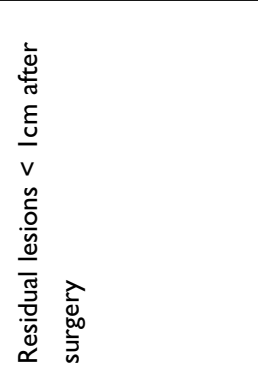 \\
\hline$\frac{8}{\stackrel{8}{0}}$ & $\bar{\simeq}$ & $\stackrel{\cong}{\simeq}$ & $\stackrel{n}{\sim}$ & $\stackrel{\infty}{\stackrel{\infty}{+}}$ & 오 & $\frac{n}{\sigma}$ & $\stackrel{\tilde{\sigma}}{2}$ \\
\hline 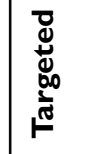 & $\Xi$ & 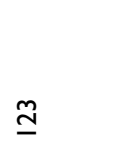 & on & $\underset{f}{\mathfrak{f}}$ & 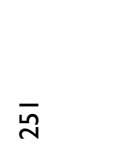 & ণ্ণ & స్ర \\
\hline 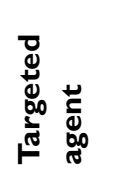 & 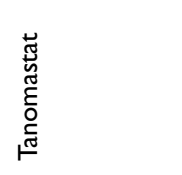 & 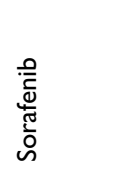 & 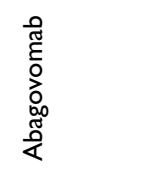 & 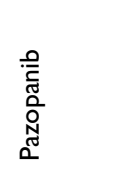 & 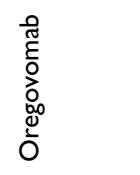 & 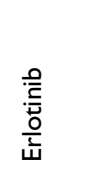 & 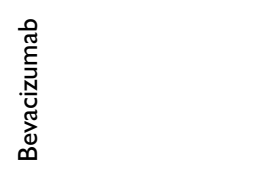 \\
\hline Oᄋ & $\stackrel{\geqq}{\equiv}$ & $\S$ & $\stackrel{\geqq}{\equiv}$ & $\stackrel{\geqq}{=}$ & $\stackrel{\stackrel{一}{\equiv}}{=}$ & $\begin{array}{l}\equiv \\
\equiv\end{array}$ & $\stackrel{\geqq}{\equiv}$ \\
\hline 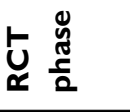 & $\equiv$ & $=$ & $\equiv$ & $\equiv$ & $\equiv$ & $\equiv$ & $\equiv$ \\
\hline 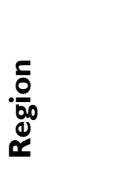 & 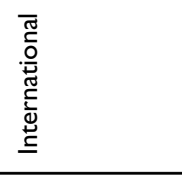 & 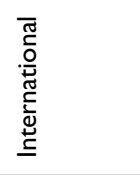 & 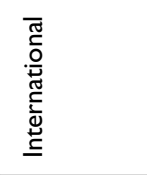 & 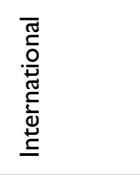 & 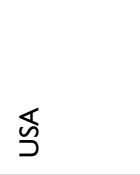 & 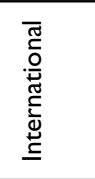 & 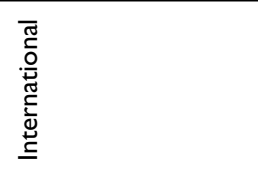 \\
\hline ঠ் & ঃั & $\frac{m}{i}$ & $\frac{m}{i}$ & $\frac{\nabla}{\circ}$ & ఫ્ণે & $\frac{\pi}{2}$ & $\overline{\bar{i}}$ \\
\hline ते & 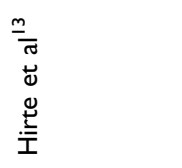 & 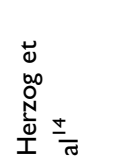 & 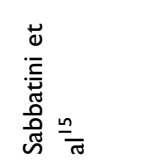 & $\begin{array}{l}\frac{a}{\pi} \\
\overrightarrow{0} \\
\frac{0}{0} \\
0\end{array}$ & 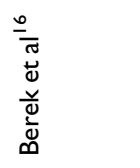 & 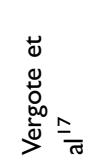 & 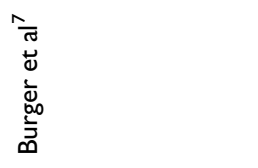 \\
\hline
\end{tabular}




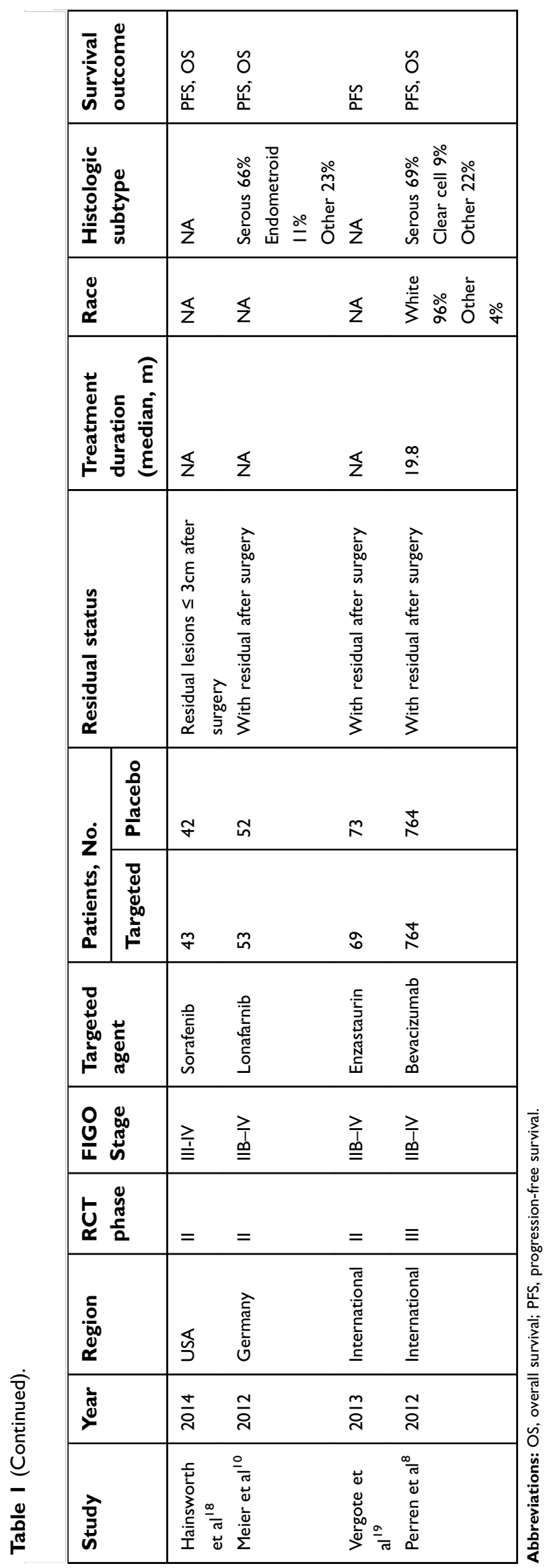



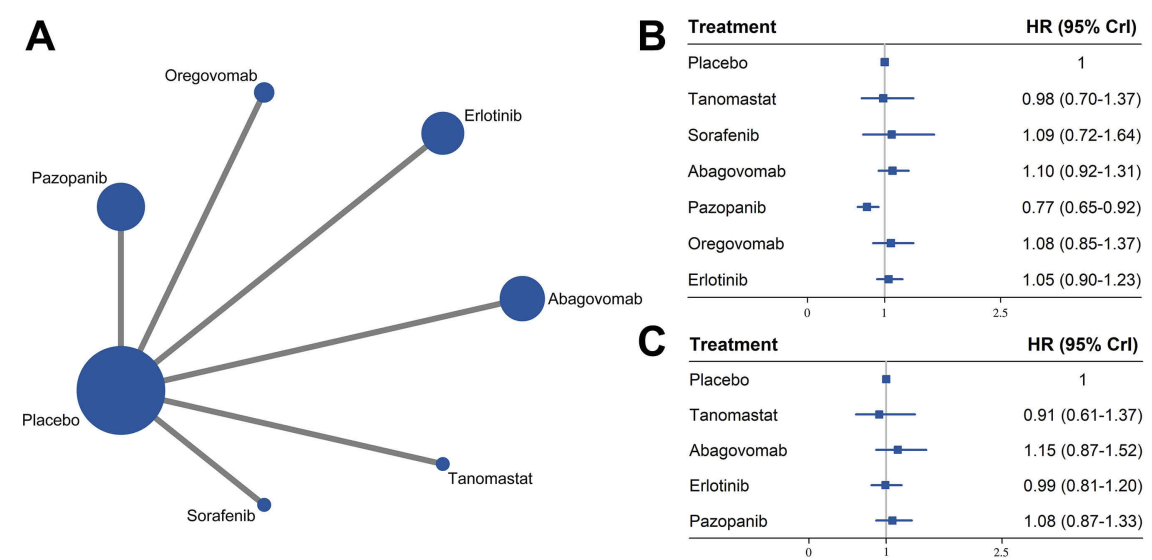

Figure 2 Results of pure targeted maintenance treatment: (A) network of eligible comparisons; (B) network meta-analysis on progression-free survival; (C) network metaanalysis on overall survival.

In terms of OS, none of the treatments (pazopanib, tanomastat, abagovomab, and erlotinib) were superior to placebo (Figure 2C).

\section{Targeted-throughout treatment}

Bevacizumab, sorafenib, lonafarnib, and enzastaurin were represented in our network meta-analysis of targetedthroughout treatment (Figure 3A). Patients who received bevacizumab demonstrated an advantage in PFS compared with placebo (HR, 0.76; 95\% CrI, 0.69-0.84); the remaining targeted-throughout regimens were not associated with an improved PFS (Figure 3B). Regarding OS, none of the intervention measures resulted in a significant improvement in survival compared with placebo (Figure 3C).

\section{Pure targeted maintenance versus targeted-} throughout treatment

In order to detect the effectiveness of targeted therapy in the first-line chemotherapy phase, we conducted an analysis grouping together the pure targeted maintenance studies or the targeted-throughout treatment studies and comparing them with placebo (Figure 4A). For PFS, pooled HRs showed an advantage with targeted-throughout treatment compared with placebo (HR, 0.79, 95\% CrI, 0.67-0.97, Figure 4B). However, there was no significant difference between targeted-throughout and pure targeted maintenance treatment (HR, 0.80; 95\% CrI, 0.64-1.03). For OS, targetedthroughout treatment had no superiority to pure targeted maintenance treatment (HR, 0.92; 95\% CrI, 0.69-1.21, Figure 4C); this result paralleled the results for PFS.

\section{Pazopanib versus bevacizumab}

It is widely known that the use of bevacizumab in first-line chemotherapy remains controversial. However, no RCTs have been conducted comparing bevacizumab in pure maintenance treatment and bevacizumab-throughout treatment. Thus, we compared bevacizumab-throughout treatment and pure pazopanib maintenance treatment (Figure 5A). Pazopanib is an inhibitor of the VEGF pathway and thus is similar to bevacizumab. The results indicated that
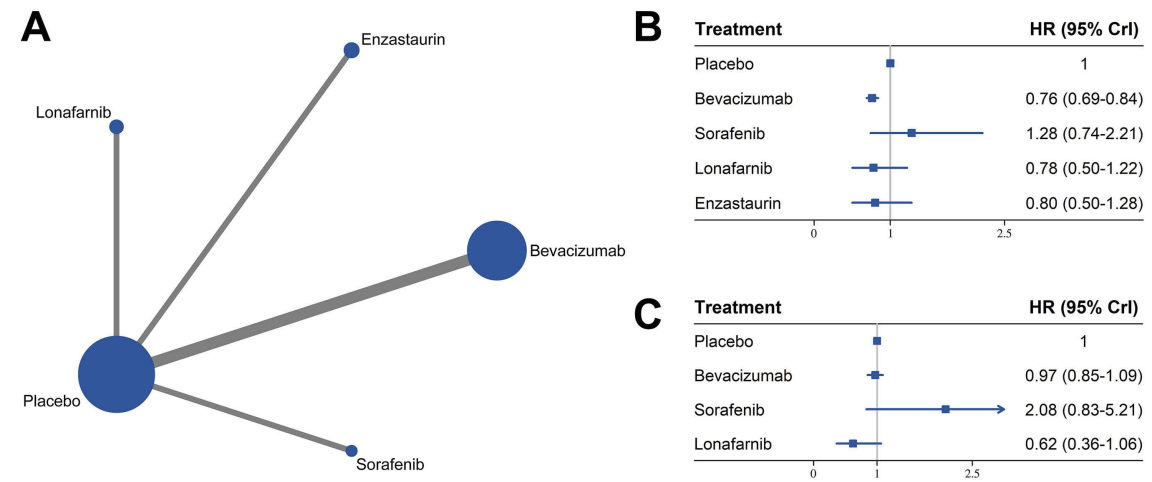

Figure 3 Results of targeted-throughout treatment: (A) network of eligible comparisons; (B) network meta-analysis on progression-free survival; (C) network meta-analysis on overall survival. 
A

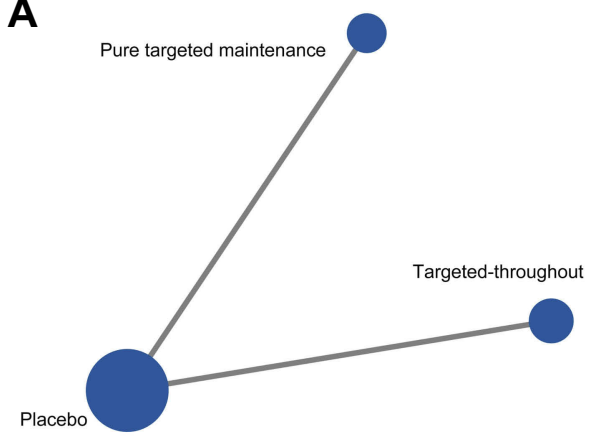

B

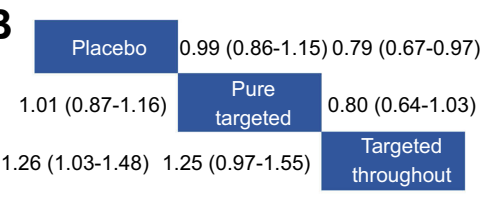

C

Placebo $1.04(0.86-1.26) \quad 0.95(0.77-1.17)$

$0.96(0.79-1.17) \quad \begin{gathered}\text { Pure } \\ \text { targeted }\end{gathered} 0.92(0.69-1.21)$

$1.05(0.86-1.30) 1.09(0.83-1.46) \quad \begin{gathered}\text { Targeted } \\ \text { throughout }\end{gathered}$

Figure 4 Comparisons between pure targeted maintenance and targeted-throughout treatment: (A) network of eligible comparisons; (B) network meta-analysis on progression-free survival; (C) network meta-analysis on overall survival.
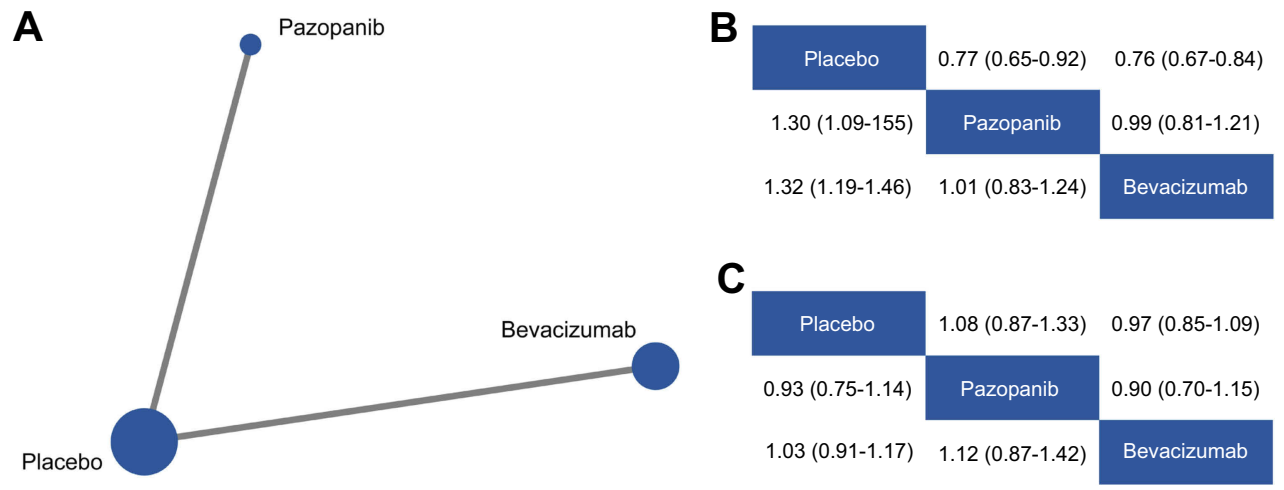

Figure 5 Comparisons between pazopanib and bevacizumab: (A) network of eligible comparisons; (B) network meta-analysis on progression-free survival; (C) network meta-analysis on overall survival.

pazopanib and bevacizumab shared equivalent efficacy, and there were no significant differences for all treatment outcomes (PFS: HR, 0.99; 95\% CrI, 0.81-1.21 and OS: HR, 0.89; 95\% CrI, 0.70-1.15) (Figure 5B and C).

\section{Quality of evidence}

No severe risk of bias was found among the majority of studies using the Cochrane risk of bias tool. The risk of bias summary by domain is summarized in Figure S1.

We evaluated the consistency of network meta-analysis by comparison of the HRs resulting from direct analyses with the corresponding results from indirect analyses. The results are shown in Table S1; results were similar between pairwise and indirect comparisons, suggesting consistency within the model.

\section{Publication bias}

Begg's test was applied to assess publication bias. The results of Begg's funnel plots did not reveal any evidence of obvious asymmetry for OS (Figure $\mathrm{S} 2, P=0.805$ ) or PFS
(Figure $\mathrm{S} 3, P=0.484$ ). Sensitivity analysis indicated that results for PFS and OS were robust (Figures S4 and S5).

\section{Discussion}

Multiple evidence-based targeted maintenance treatments have been investigated in trials of newly diagnosed ovarian cancer. However, the comparison of these regimens has remained unclear. We constructed a network metaanalysis including 11 RCTs to evaluate the effect of nine targeted regimens for maintenance therapy. The results showed that pazopanib-pure maintenance therapy and bevacizumab-throughout treatment resulted in significantly better PFS for newly diagnosed ovarian cancer patients. In addition, there did not appear to be any difference between pazopanib-pure maintenance and bevacizumabthroughout treatment in terms of survival.

The prognosis for advanced ovarian cancer patients is poor. Although treatment consisting of surgical debulking and platinum-taxane-based chemotherapy is widely used, it has been reported that more than $80 \%$ of patients experience disease recurrence which eventually becomes the 
main cause of their death. ${ }^{26}$ Maintenance therapy is one strategy that has garnered clinical attention and academic debate. In theory, maintenance agents could inhibit proliferation of the resistant cancer cell subpopulations after front-line chemotherapy and sequentially delay disease progression. Unfortunately, there is no convincing evidence that maintenance therapy with cytotoxic agents can confer a long-term survival advantage, in fact, evidence points to a high-risk of increased toxicity with these agents. ${ }^{6,27,28}$ At present, maintenance therapy with cytotoxic agents is not recommended. ${ }^{29}$

Given the disappointing findings from conventional agents, target maintenance agents, which have different growth inhibiting properties than conventional cytotoxic drugs, are rapidly emerging in ovarian cancer treatment. Moreover, trials tend to use one of two therapeutic strategies for targeted maintenance therapy: with or without targeted therapy at the first-line chemotherapy phase. To determine the effectiveness of a targeted regimen in maintenance therapy, we separately analyzed the pure targeted maintenance group and the targeted-throughout group. We cannot rule out the possibility that exposure to targeted drugs during chemotherapy contributed to the observed survival improvement. Our results showed that improved PFS was observed for pazopanib and bevacizumab in the pure targeted maintenance group and the targeted-throughout group, respectively. Pazopanib is a tyrosine kinase inhibitor of VEGFR and bevacizumab is anti-VEGF monoclonal antibody. Thus, the results of our meta-analysis suggest that VEGF and angiogenesis are important promoters of ovarian cancer progression. No PFS or OS benefit was observed with abagovomab or oregovomab, both of which are monoclonal antibodies specific for CA125. Anti-CA-125 monoclonal antibody maintenance treatment might not be effective for newly diagnosed ovarian cancer. With low expression in other tissue sites and the modulation of proliferation and invasiveness in ovarian cancer, CA-125 (MUC16) is ideally suited for targeting. Recently, studies have shown that $\mathrm{T}$ cells modified to express a chimeric antigen receptor specific to MUC16 can kill ovarian cancer cells, ${ }^{30}$ indicating that anti-CA-125 monoclonal antibody may be used in combination with immunotherapy in the future.

Currently, the timing of targeted therapy is quite controversial, particularly, it is widely debated as to whether it should be used throughout first-line chemotherapy and maintenance periods or only in the maintenance period. Our results indicated that targeted-throughout treatment was not superior to pure targeted maintenance treatment. These findings suggest that target therapy plays a real role in delaying disease progression during the maintenance stage. The GOG-0218 trial compared chemotherapy plus bevacizumab-initiation, chemotherapy plus bevacizumabthroughout, and chemotherapy alone with placebo. ${ }^{7}$ The results demonstrated that bevacizumab-throughout improved PFS, whereas bevacizumab-initiation showed no significant survival improvement compared with chemotherapy alone. At present, there are no well-designed RCTs examining pure bevacizumab maintenance therapy for newly diagnosed ovarian cancer. To determine the effectiveness of bevacizumab in the maintenance treatment period, we compared bevacizumab-throughout treatment and pure pazopanib maintenance treatment. Both drugs inhibit the VEGF pathway. The results showed no significant different between bevacizumab-throughout and pure pazopanib maintenance treatment for PFS or OS. These results showed that the effect of bevacizumab maintenance alone may be similar to that of bevacizumab-throughout treatment. The addition of bevacizumab to the treatment regimen of patients with newly diagnosed chemotherapy should be considered. Future trials should properly evaluate bevacizumab-throughout and pure bevacizumab maintenance treatment.

It was recently reported that maintenance therapy with either olaparib or niraparib, poly (ADP-ribose) polymerase (PARP) inhibitors, significantly improved survival for patients with platinum-sensitive, recurrent ovarian cancer compared with the matching placebo. ${ }^{31,32}$ We did not incorporate these two drugs into this analysis because of the heterogeneity between recurrent and newly diagnosed tumors and because PARP inhibitors cause the formation of double-strand DNA breaks that cannot be repaired, thus leading to cell death. This approach of tumor inhibition is completely different from that of VEGF inhibitors. There is currently insufficient evidence to evaluate the efficacy of PARP inhibitors as maintenance therapy for newly diagnosed ovarian cancer. It remains to be seen whether consistent results could be obtained in newly diagnosed ovarian cancer.

In the AGO OVAR 16 trial, pazopanib as maintenance therapy was reported to improve survival for East Asian patients, but the benefit was limited in the non-East Asian group. ${ }^{9}$ In addition, stratified analysis of the GOG-218 study showed that patients with a high risk of progression (stage IV disease, inoperable stage III disease, or suboptimally debulked stage III disease) obtained a longer 
survival advantage compared with those who received bevacizumab maintenance therapy. ${ }^{33}$ These results suggest a need to identify biomarkers and clinicopathologic characteristics to select patients with ovarian cancer for targeted maintenance therapy.

Several limitations should be acknowledged in the present study. Our meta-analysis showed that although targeted maintenance treatment demonstrated an improvement in PFS, it did not lead to an OS benefit. Notably, PFS is the preferred end point for newly diagnosed cancer in the majority of included trials, which is in-line with recommendations from the Gynecologic Cancer Intergroup. ${ }^{34}$ In addition, the confounding effect of post-progression therapy is inescapable for OS. Heterogeneity among patients existed; patients received different durations of targeted therapy and the majority of included patients were Caucasian, although Asians made up a portion of the study population in some reports. Serious carcinoma was the main pathological type of cancer so the benefits of targeted maintenance treatment for other pathological types need to be considered. Taking these limitations into account, caution should be taken with the clinical application of our results. Tumor residual status and pathologic/clinical complete response were important prognostic covariates, but there was insufficient data for stratified analyses. We searched Medline, Embase, and the Cochrane Library databases in our study. To our knowledge, studies reporting significant findings were more likely to be published in English language journals, whereas negative results were almost exclusively published in native language journals. Native language journals were difficult to obtain and thus were excluded from our analysis. In addition to this, there are many ongoing studies on targeted therapy for ovarian cancer the results of which may have implications for our results.

\section{Conclusion}

In conclusion, our findings showed a potential survival advantage conferred by pazopanib and bevacizumabthroughout as maintenance therapy for newly diagnosed ovarian cancer patients. Clinical trials with a direct headto-head comparison are needed to examine the effectiveness of bevacizumab maintenance alone and to identify the specific subgroup(s) that will benefit from this therapeutic approach.

\section{Acknowledgments}

We thank Sarah Bubeck, Ph.D., from Liwen Bianji, Edanz Editing China (www.liwenbianji.cn/ac), for editing the
English text of a draft of this manuscript. This work was supported by National Natural Science Foundation of China (No.81872441) and Natural Science Foundation of Guangdong Province (No.2018A030313735).

\section{Author contributions}

All authors contributed to data analysis, drafting or revising the article, gave final approval of the version to be published, and agree to be accountable for all aspects of the work.

\section{Disclosure}

The authors report no conflicts of interest in this work.

\section{References}

1. Bray F, Ferlay J, Soerjomataram I, Siegel RL, Torre LA, Jemal A. Global cancer statistics 2018: GLOBOCAN estimates of incidence and mortality worldwide for 36 cancers in 185 countries. CA Cancer J Clin. 2018;68(6):394-424. doi: 10.3322/caac.21492.

2. Thigpen T, duBois A, McAlpine J, et al. First-line therapy in ovarian cancer trials. Int $J$ Gynecol Cancer. 2011;21(4):756-762. doi:10.1097/IGC.0b013e31821ce75d

3. Cannistra SA. Cancer of the ovary. $N$ Engl J Med. 2004;351 (24):2519-2529. doi:10.1056/NEJMra041842

4. De Placido S, Scambia G, Di Vagno G, et al. Topotecan compared with no therapy after response to surgery and carboplatin/paclitaxel in patients with ovarian cancer: multicenter Italian Trials in Ovarian Cancer (MITO-1) randomized study. J Clin Oncol. 2004;22 (13):2635-2642. doi:10.1200/JCO.2004.09.088

5. Pfisterer J, Weber B, Reuss A, et al. Randomized phase III trial of topotecan following carboplatin and paclitaxel in first-line treatment of advanced ovarian cancer: a gynecologic cancer intergroup trial of the AGO-OVAR and GINECO. J Natl Cancer Inst. 2006;98 (15):1036-1045. doi:10.1093/jnci/djj296

6. Pecorelli S, Favalli G, Gadducci A, et al. Phase III trial of observation versus six courses of paclitaxel in patients with advanced epithelial ovarian cancer in complete response after six courses of paclitaxel/platinum-based chemotherapy: final results of the After-6 protocol 1. J Clin Oncol. 2009;27(28):4642-4648. doi:10.1200/ JCO.2009.21.9691

7. Burger RA, Brady MF, Bookman MA, et al. Incorporation of bevacizumab in the primary treatment of ovarian cancer. $N$ Engl $J$ Med. 2011;365(26):2473-2483. doi:10.1056/NEJMoa1104390

8. Perren TJ, Swart AM, Pfisterer J, et al. A phase 3 trial of bevacizumab in ovarian cancer. $N$ Engl J Med. 2011;365(26):2484-2496.

9. Du Bois A, Floquet A, Kim JW, et al. Incorporation of pazopanib in maintenance therapy of ovarian cancer. J Clin Oncol. 2014;32 (30):3374-3382. doi:10.1200/JCO.2014.55.7348

10. Meier W, Du Bois A, Rau J, et al. Randomized phase II trial of carboplatin and paclitaxel with or without lonafarnib in first-line treatment of epithelial ovarian cancer stage IIB-IV. Gynecol Oncol. 2012;126(2):236-240. doi:10.1016/j.ygyno.2012.04.050

11. Korkmaz T, Seber S, Basaran G. Review of the current role of targeted therapies as maintenance therapies in first and second line treatment of epithelial ovarian cancer; In the light of completed trials. Crit Rev Oncol Hematol. 2016;98:180-188. doi:10.1016/j. critrevonc.2015.10.006

12. Qian X, Qin J, Pan S, Li X, Pan Y, Ma S. Maintenance therapy in ovarian cancer with targeted agents improves PFS and OS: a systematic review and meta-analysis. PLoS One. 2015;10(9):e0139026. doi:10.1371/journal.pone.0117702 
13. Cipriani A, Higgins JP, Geddes JR, Salanti G. Conceptual and technical challenges in network meta-analysis. Ann Intern Med. 2013;159 (2):130-137. doi:10.7326/0003-4819-159-2-201307160-00008

14. Salanti G. Indirect and mixed-treatment comparison, network, or multiple-treatments meta-analysis: many names, many benefits, many concerns for the next generation evidence synthesis tool. Res Synth Methods. 2012;3(2):80-97. doi:10.1002/jrsm.1037

15. Group G, Paoletti X, Oba K, et al. Benefit of adjuvant chemotherapy for resectable gastric cancer: a meta-analysis. Jama. 2010;303 (17):1729-1737. doi:10.1001/jama.2010.534

16. van Beurden-Tan CHY, Franken MG, Blommestein HM, Uyl-de Groot CA, Sonneveld P. Systematic literature review and network meta-analysis of treatment outcomes in relapsed and/or refractory multiple Myeloma. J Clin Oncol. 2017;35(12):1312-1319. doi:10.1200/JCO.2016.71.1663

17. Parmar MK, Torri V, Stewart L. Extracting summary statistics to perform meta-analyses of the published literature for survival endpoints. Stat Med. 1998;17(24):2815-2834.

18. Gelman A, Rubin DB. Markov chain Monte Carlo methods in biostatistics. Stat Methods Med Res. 1996;5(4):339-355. doi:10.1177/ 096228029600500402

19. Hirte H, Vergote IB, Jeffrey JR, et al. A phase III randomized trial of BAY 12-9566 (tanomastat) as maintenance therapy in patients with advanced ovarian cancer responsive to primary surgery and paclitaxel/platinum containing chemotherapy: a National Cancer Institute of Canada Clinical Trials Group Study. Gynecol Oncol. 2006;102 (2):300-308. doi:10.1016/j.ygyno.2005.12.020

20. Herzog TJ, Scambia G, Kim BG, et al. A randomized phase II trial of maintenance therapy with Sorafenib in front-line ovarian carcinoma. Gynecol Oncol. 2013;130(1):25-30. doi:10.1016/j.ygyno. 2013.04.011

21. Sabbatini P, Harter P, Scambia G, et al. Abagovomab as maintenance therapy in patients with epithelial ovarian cancer: a phase III trial of the AGO OVAR, COGI, GINECO, and GEICO-the MIMOSA study. $J$ Clin Oncol. 2013;31(12):1554-1561. doi:10.1200/JCO.2012.46.4057

22. Berek J, Taylor P, McGuire W, Smith LM, Schultes B, Nicodemus CF. Oregovomab maintenance monoimmunotherapy does not improve outcomes in advanced ovarian cancer. $J$ Clin Oncol. 2009;27(3):418-425. doi:10.1200/JCO.2008.17.8400

23. Vergote IB, Jimeno A, Joly F, et al. Randomized phase III study of erlotinib versus observation in patients with no evidence of disease progression after first-line platin-based chemotherapy for ovarian carcinoma: a European Organisation for Research and Treatment of Cancer-Gynaecological Cancer Group, and gynecologic cancer intergroup study. J Clin Oncol. 2014;32(4):320-326. doi:10.1200/ JCO.2013.50.5669
24. Hainsworth JD, Thompson DS, Bismayer JA, et al. Paclitaxel/carboplatin with or without sorafenib in the first-line treatment of patients with stage III/IV epithelial ovarian cancer: a randomized phase II study of the Sarah Cannon Research Institute. Cancer Med. 2015;4 (5):673-681. doi:10.1002/cam4.376

25. Vergote IB, Chekerov R, Amant F, et al. Randomized, phase II, placebo-controlled, double-blind study with and without enzastaurin in combination with paclitaxel and carboplatin as first-line treatment followed by maintenance treatment in advanced ovarian cancer. $J$ Clin Oncol. 2013;31(25):3127-3132. doi:10.1200/JCO.2012.44.9116

26. Martin LP, Schilder RJ. Management of recurrent ovarian carcinoma: current status and future directions. Semin Oncol. 2009;36(2):112125. doi:10.1053/j.seminoncol.2008.12.003

27. Mannel RS, Brady MF, Kohn EC, et al. A randomized phase III trial of IV carboplatin and paclitaxel $x 3$ courses followed by observation versus weekly maintenance low-dose paclitaxel in patients with early-stage ovarian carcinoma: a Gynecologic Oncology Group Study. Gynecol Oncol. 2011;122(1):89-94. doi:10.1016/j.ygyno.2011.03.013

28. Mei L, Chen H, Wei DM, et al. Maintenance chemotherapy for ovarian cancer. Cochrane Database Syst Rev. 2013;29(6):CD007414.

29. Bookman MA. Optimal primary therapy of ovarian cancer. Ann Oncol. 2016;27(Suppl 1):i58-i62. doi:10.1093/annonc/mdw088

30. Chekmasova AA, Brentjens RJ. Adoptive T cell immunotherapy strategies for the treatment of patients with ovarian cancer. Discov Med. 2010;9(44):62-70.

31. Ledermann J, Harter P, Gourley C, et al. Olaparib maintenance therapy in platinum-sensitive relapsed ovarian cancer. $N$ Engl J Med. 2012;366(15):1382-1392. doi:10.1056/NEJMoa1105535

32. Mirza MR, Monk BJ, Herrstedt J, et al. Niraparib maintenance therapy in platinum-sensitive, recurrent ovarian cancer. $N$ Engl J Med. 2016;375(22):2154-2164. doi:10.1056/NEJMoa1611310

33. Oza AM, Cook AD, Pfisterer J, et al. Standard chemotherapy with or without bevacizumab for women with newly diagnosed ovarian cancer (ICON7): overall survival results of a phase 3 randomised trial. Lancet Oncol. 2015;16(8):928-936. doi:10.1016/S1470-2045(15) 00086-8

34. Stuart GC, Kitchener H, Bacon M, et al. 2010 Gynecologic Cancer InterGroup (GCIG) consensus statement on clinical trials in ovarian cancer: report from the fourth ovarian cancer consensus conference. Int $J$ Gynecol Cancer. 2011;21(4):750-755. doi:10.1097/ IGC.0b013e31821b2568
Cancer Management and Research is an international, peer-reviewed open access journal focusing on cancer research and the optimal use of preventative and integrated treatment interventions to achieve improved outcomes, enhanced survival and quality of life for the cancer patient.
The manuscript management system is completely online and includes a very quick and fair peer-review system, which is all easy to use. Visit http://www.dovepress.com/testimonials.php to read real quotes from published authors. 